\title{
Primeiro registro da família Furipteridae (Mammalia, Chiroptera) para o Estado do Rio de Janeiro, Brasil
}

\author{
André Pol ${ }^{1}$, Marcelo R. Nogueira ${ }^{1} \&$ Adriano L. Peracchi ${ }^{2}$ \\ 1 Programa de Pós-graduação em Biologia Animal, Universidade Federal Rural do Rio de Janeiro. 23890-000 Seropédica, Rio \\ de Janeiro, Brasil. E-mail: andrepol@infolink.com.br; mrnogueira@rionet.com.br \\ 2 Departamento de Biologia Animal, Instituto de Biologia, Universidade Federal Rural do Rio de Janeiro. Caixa Postal 74503, \\ 23851-970 Seropédica, Rio de Janeiro, Brasil. E-mail: aperacchi@webdigital.com.br
}

\begin{abstract}
First record of the family Furipteridae (Mammalia, Chiroptera) for the state of Rio de Janeiro, Brazil. Furipterus horrens (Cuvier, 1828), the only species in the family Furipteridae known to occur in the Brazilian territory, is reported for the first time in the state of Rio de Janeiro. The single specimen obtained was found alone in its diurnal roost in a cavity formed among large granite blocks in a well-preserved Atlantic Forest area. Morphometric data obtained from this specimen are within the known range documented for the species.
\end{abstract}

KEY WORDS. Distribution, Furipterus horrens, southeastern Brazil.

A família Furipteridae é composta por dois gêneros monotípicos (Amorphochilus Peters, 1877 e Furipterus Bonaparte, 1837) e encontra-se representada no Brasil apenas por Furipterus horrens (Cuvier, 1828) (Koopman 1993). Os membros dessa família são estritamente neotropicais e facilmente reconhecidos por sua anatomia peculiar. Em ambos os gêneros o polegar apresentase bastante reduzido e incluído na membrana alar até a base da unha, que é pequena e não funcional (NowAK 1994). A presença de mamas abdominais com função lactífera é também reportada para essa família (UIEDA et al. 1980), embora SimMONS (1993) não as tenha observado nas duas fêmeas adultas de $F$. horrens incluídas em seu estudo.

Furipterus horrens é um morcego de pequeno porte ( $c a .3$ $\mathrm{g})$, estritamente insetívoro, e que tem sido registrado abrigandose em cavernas, ocos de árvore, e dentro ou sob árvores caídas em vários estágios de decomposição (UIEDA et al. 1980, SIMMONs \& Voss 1998). Sua distribuição geográfica estende-se da Costa Rica até o Peru e sul do Brasil (até Santa Catarina), incluindo Trinidad (Vieira 1942, Koopman 1993). Parece ocorrer preferencialmente em associação a formações vegetais úmidas (Handley 1976, Uieda et al. 1980). No Brasil sua ocorrência é conhecida por registros esparsos nas regiões norte (PICcININI 1974, Pinto-Da-Rocha 1995, Voss \& Emmons 1996), nordeste (Vieira 1942, Piccinini 1974, Willig \& Mares 1989, Silva et al. 2001), sudeste (Trajano 1984, Pinto-Da-Rocha 1995, Portfors et. al. 2000), centro-oeste (CoImbra et al. 1982, BREDT et al. 1999) e sul (VIEIRA 1942).
Em 3/I/2001, durante visita à Área de Proteção Ambiental (APA) de Cairuçu, município de Parati, região litorânea no extremo sul do Estado do Rio de Janeiro, sudeste do Brasil, tivemos oportunidade de colecionar um exemplar de $F$. horrens, cuja ocorrência era ainda desconhecida para essa unidade federativa. Em sua porção continental, a APA-Cairuçu estendese por uma área de 33.800 ha, situando-se na vertente oceânica da Serra do Mar (MARQUEs 1997). A formação vegetal dominante na região é a Floresta Ombrófila Densa, que na localidade de coleta sofre forte influência marinha chegando próximo à estreita faixa arenosa da praia (MARQues 1997). O clima é classificado como mesotérmico, superúmido, com pouco ou nenhum déficit hídrico (MArques 1997).

O exemplar em questão é um macho adulto capturado com a mão quando em repouso em abrigo diurno (teto de cavidade formada por matacões de granito) situado na encosta íngreme do sítio denominado localmente de "Praia da

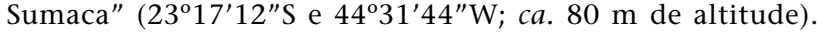
Apesar de um morador da localidade ter relatado a presença de grande número de morcegos na cavidade onde foi encontrado o exemplar, no momento da captura nenhum outro morcego foi observado. Durante estudo conduzido nas florestas de Paracou, Guiana Francesa, Simmons \& Voss (1998) encontraram $F$. horrens em nove abrigos diurnos, nenhum dos quais ocupado por mais de um indivíduo adulto (macho ou fêmea). Grupos de dois indivíduos foram encontrados, mas estes eram compostos por uma fêmea lactante e seu filho- 
te (Simmons \& Voss 1998). Em outros refúgios localizados no sudeste brasileiro, como os reportados por Trajano (1984) em estudo de populações cavernícolas do Vale do Ribeira, Estado de São Paulo, indivíduos isolados ou pequenos agrupamentos de no máximo três indivíduos também foram observados. Contudo, F. horrens pode formar também colônias bastante numerosas (150 a 250 indivíduos), como observado por Uieda et al. (1980) em grutas no Ceará. Estes autores sugeriram que tal variação no tamanho das colônias pode estar relacionada às dimensões dos locais de abrigo, mesma suposição levantada por MARQues (1985) e Nogueira \& Pol (1998) em relação a colônias de Carollia perspicillata (Linnaeus, 1758) e Rhynchonycteris naso (Wied-Neuwied, 1820), respectivamente.

O exemplar aqui reportado encontra-se preservado em álcool 70\% (crânio removido) e foi incorporado, sob o código ALP5932, à Coleção Adriano Lúcio Peracchi, depositada no Instituto de Biologia da Universidade Federal Rural do Rio de Janeiro. Este espécime confere em todos os aspectos com a diagnose fornecida para a espécie por Husson $(1962,1978)$ e sua morfometria encontra-se dentro da variação atualmente conhecida para a espécie (Husson 1962, 1978, BRosset \& CHARlesDominique 1990, Simmons \& Voss 1998). Medidas (em mm) selecionadas, obtidas de acordo com o protocolo apresentado por Simmons \& Voss (1998), são como segue: antebraço, 37.9; comprimento total do crânio, 12.1; comprimento côndilo-basal, 11.4; largura mastóidea, 6.4; largura da caixa craniana, 5.8; constricção pós-orbital, 3.0; largura através dos molares superiores, 4.7 .

A ausência de $F$. horrens em inventários conduzidos até então no Estado do Rio de Janeiro não constitui indício consistente de que a espécie não mais ocorra nessas áreas. Como ressaltado por Nogueira et al. (2002) em recente registro de outro morcego insetívoro aéreo (Saccopteryx leptura (Schreber, 1774), Emballonuridae) para o Estado do Rio de Janeiro, áreas como o Maciço da Tijuca, no município do Rio de Janeiro, foram intensivamente amostradas, mas apenas com o uso de redes de neblina armadas ao nível do solo (Esbérard 1998). Dados obtidos por Simmons \& Voss (1998) evidenciam a importância da busca ativa de abrigos para a amostragem de F. horrens. Dos 13 espécimes coletados por esses autores, 12 foram obtidos em abrigos diurnos e um abatido a tiro quando em vôo noturno. BREDT et al. (1999) destacaram ainda que redes de neblina se mostraram ineficientes na captura de $F$. horrens mesmo quando armadas na entrada ou no interior das cavernas (redes entomológicas foram usadas com sucesso). $\mathrm{O}$ uso de armadilhas do tipo harpa ("harp traps"), que pode apresentar eficiência na captura de espécies não susceptíveis às redes de neblina (Kunz \& KunTA 1988), mostrou-se também satisfatório na captura de $F$. horrens em abrigos diurnos no Parque Estadual Intervales, sul do Estado de São Paulo (F.C. Passos comunicação pessoal). Além de propiciar um maior conhecimento sobre a biologia dessa espécie (Simmons \& Voss 1998), a identificação de abrigos diurnos constitui um importante método complementar na amostragem das comunidades de morcegos neotropicais (Voss \& EMmons 1996, Simmons \& Voss 1998, PorTfors et. al. 2000).

\section{AGRADECIMENTOS}

Os autores agradecem à CAPES e ao CNPq pelas bolsas concedidas.

\section{REFERÊNCIAS BIBLIOGRÁFICAS}

Bredt, A.; W. Uieda \& E.D. Magalhães. 1999. Morcegos cavernícolas da região do Distrito Federal, centro-oeste do Brasil (Mammalia, Chiroptera). Revista Brasileira de Zoologia, Curitiba, 16 (3): 731-770.

Brosset, A. \& P. Charles-Dominique. 1990. The bats from French Guiana: a taxonomic, faunistic and ecological approach. Mammalia, Paris, 54 (4): 509-560.

Coimbra Jr., C.E.A.; M.M. Borges; D.Q. Guerra \& D.A. Mello. 1982. Contribuição à zoogeografia e ecologia de morcegos em regiões de cerrado do Brasil Central. Boletim Técnico da Revista Brasil Florestal, Brasília, 7: 34-38.

EsBÉRARD, C.E.L. 1998. Validade dos parâmetros da IUCN em amostra regional - há espécies de morcegos ameaçadas no município do Rio de Janeiro? Boletim FBCN, Rio de Janeiro, 25: 71-86.

Handley JR., C.O. 1976. Mammals of the Smithsonian Venezuelan project. Brigham Young University Science Bulletin, Provo, 20 (5): 1-91.

Husson, A.M. 1962. The bats of Suriname. Zoologische Verhandelingen., Leiden, 58: 1-282.

. 1978. The mammals of Surinam. Zoölogishe Monographieën van het Rijksumuseum van Natuurlijke Historie, Leiden, 2: XXIV+1-569.

Koopman, K.F. 1993. Order Chiroptera, p. 137-241. In: D.E. WILSON \& D.M. REEDER (Eds). Mammal species of the world: a taxonomic and geographic reference. Washington, D.C., Smithsonian Institution Press, $2^{\text {nd }}$ ed., XVIII+1-1206.

Kunz, T.H. \& A. KuRTA. 1988. Capture methods and holding devices, p. 1-28. In: T.H. Kunz (Ed.). Ecological and behavioural methods for the study of bats. Washington, DC., Smithsonian Institution Press, XXII+533p.

MARQUes, S.A. 1985. Espécies associadas e algumas características físicas influindo na presença de Carollia perspicillata em bueiros na região de Manaus, AM (Mammalia, Chiroptera: Phyllostomidae). Acta Amazonica, Manaus, 15 (1-2): 243248.

Marques, M.C.M. 1997. Mapeamento da cobertura vegetal e listagem das espécies ocorrentes na Área de Proteção Ambiental de Cairuçú, município de Paratí, RJ. Série Estudos e Contribuições, Rio de Janeiro, 13: 1-96.

Nogueira, M.R. \& A. Pol. 1998. Observações sobre os hábitos de Rhynchonycteris naso (Wied-Neuwied, 1820) e Noctilio albiventris Desmarest, 1818 (Mammalia, Chiroptera). Revista Brasileira de Biologia, Rio de Janeiro, 58 (3): 473-480.

Nogueira, M.R.; A.L. Peracchi \& A. Pol. 2002. Notes on the lesser white-lined bat, Saccopteryx leptura (Schreber) (Chiroptera, Emballonuridae) from southeastern Brazil. Revista Brasileira de Zoologia, Curitiba, 19 (4): 1123-1130.

NowAK, R.M. 1994. Walker's bats of the world. Baltimore, Johns Hopkins University Press, 287p.

Piccinini, R.S. 1974. Lista provisória dos quirópteros da coleção do Museu Paraense Emílio Goeldi (Chiroptera). Boletim do Museu Paraense Emílio Goeldi, Nova Série Zoologia, Belém, 77: 1-32.

PinTo-DA-Rocha, R. 1995. Sinopse da fauna cavernícola do Brasil (1907-1994). Papéis Avulsos Zoologia, São Paulo, 39 (6): 61-173. 
Portfors, C.V.; M.B. Fenton; L.M.S. Aguiar; J.E. Baumgarten; M.J. Vonhof; S. Bouchard; D.M. Faria; W.A. Pedro; N.I.L. Rauntenbach \& M. ZorTéa. 2000. Bats from Fazenda Intervales, Southeastern Brazil - species account and comparison between different sampling methods. Revista Brasileira de Zoologia, Curitiba, 17 (2): 533-538.

Silva, S.S.P.; P.G. Guedes \& A.L. Peracchi. 2001. Levantamento preliminar dos morcegos do Parque Nacional de Ubajara (Mammalia, Chiroptera), Ceará, Brasil. Revista Brasileira de Zoologia, Curitiba, 18 (1): 139-144.

Simmons, N.B. 1993. Morphology, function, and phylogenetic significance of pubic nipples in bats (Mammalia: Chiroptera). American Museum Novitates, New York, 3077: 1-37.

Simmons, N.B. \& R.S. Voss. 1998. The Mammals of Paracou, French Guiana: a Neotropical lowland rainforest fauna. Part 1. Bats. Bulletin of the American Museum of Natural
History, New York, 237: 1-219.

Trajano, E. 1984. Ecologia de populações de morcegos cavernícolas em uma região cárstica do Sudeste do Brasil. Revista Brasileira de Zoologia, Curitiba, 2 (5): 255-320.

Uieda, W.; I. Sazima \& A. Storti-Filho. 1980. Aspectos da biologia do morcego Furipterus horrens. Revista Brasileira de Biologia, Rio de Janeiro, 40 (1): 59-66.

VIEIRA, C.C. 1942. Ensaio monográfico sobre os quirópteros do Brasil. Arquivos de Zoologia, São Paulo, 3 (8): 219-471.

Voss, R.S. \& L.H. Emmons. 1996. Mammalian diversity in neotropical lowland rainforests: a preliminary assessment. Bulletin of the American Museum of Natural History, New York, 230: 1-115.

Willig, M.R. \& M.A. Mares. 1989. Mammals from the caatinga: an updated list and summary of recent research. Revista Brasileira de Biologia, Rio de Janeiro, 49 (2): 361-367.

Recebido em 13.II.2003; aceito em 21.VIII.2003. 